\title{
Negative image amplifier technique for performance enhancement of ultra wideband LNA
}

\author{
Kishor G Sawarkar ${ }^{1}$, Kushal Tuckley ${ }^{2}$ \\ ${ }^{1}$ Department of Electronics and Telecommunication, MCT's RGIT, University of Mumbai, India \\ ${ }^{2}$ Indian Institute of Technology Bombay, India
}

\begin{tabular}{l}
\hline Article Info \\
\hline Article history: \\
Received Dec 27, 2017 \\
Revised Aug 10, 2018 \\
Accepted Aug 31, 2018 \\
\hline
\end{tabular}

Keywords:

Cascaded LNA and pHEMT

Low noise amplifier (LNA)

Microstrip lines hybrid

microwave integrated circuit

(HMIC)

Ultra wideband (UWB)

\begin{abstract}
The paper aims at designing of two stage cascaded ultra-wideband (UWB) low noise amplifier (LNA) by using negative image amplifier technique. The objective of this article is to show the performance improvement using negative image amplifier technique and realization of negative valued lumped elements into microstrip line geometry. The innovative technique to realize the negative lumped elements are carried out by using Richard's Transformation and transmission line calculation. The AWR microwave office tool is used to obtain characteristics of UWB LNA design with hybrid microwave integrated circuit (HMIC) technology. The 2-stage cascaded LNA design using negative image amplifier technique achieves average gain of $23 \mathrm{~dB}$ gain and low noise figure of less than $2 \mathrm{~dB}$ with return loss less than $8 \mathrm{~dB}$ for UWB 3-10GHz. The Proper bias circuit is extracted using DC characteristics of transistor at biasing point $2 \mathrm{~V}, 20 \mathrm{~mA}$ and discussed in detail with LNA layout. The negative image matching technique is applied for both input and output matching network. This work will be useful for all low power UWB wireless receiver applications.
\end{abstract}

Copyright (C) 2019 Institute of Advanced Engineering and Science. All rights reserved.

\section{Corresponding Author:}

Kishor G Sawarkar,

Research Scholar at MCT's RGIT, University of Mumbai, India,

Department of Electronics and Telecommunication,

Juhu Versova, Mumbai, India.

Email: sawarkarkishor@gmail.com, kishor.sawarkar@mctrgit.ac.in

\section{INTRODUCTION}

In today's world LNA design innovation is developing rapidly in the field of electronics and telecommunication services. Since, the interest for fast advancing in multimedia communication has moved towards research a greater demand in the field of RF and Microwave engineering. In most of the LNA design techniques pHEMT and HEMT transistors provides promising results at microwave frequencies. A LNA plays the vital role in overall performance of RF receiver. It is the first component in any RF receiver. The general topology of LNA consists of three stages: input matching network, the amplifier itself and output matching network [1-6].

Recently InGaAs ba sed pHEMT have exhibited better linearity, high survivability, less noisy and better performance than GaAs FET's [7], [8]. InGaAs based pHEMTs offering less microwave noise figure with a very less Power Consumption and high transducer gain at low voltage and current. The Two critical factors in this regard are high gain and minimize gate current. Since gate current is a component contributing to shot noise, decreased leakage gate current improves the noise performance of the HEMTs [9]. Several amplifier architectures are considered which includes distributed amplifier, lumped feedback amplifier, cascode and cascade topology [10-15]. For UWB, the distributed amplifier requires more number of transistors than the lumped feedback which consume more power. The distributed amplifier likewise languishes low efficiency when designed for high gain and normally higher noise performance. In this paper 
two stage cascaded UWB LNA is designed without compromising noise figure, gain and return loss. By using the ideal negative lumped elements for input and output matching of LNA design has achieved gain more than $21 \mathrm{~dB}$, Noise Figure (NF) less than $2 \mathrm{~dB}$ and return losses less than -10dB throughout band of 3$10 \mathrm{GHz}$. The efficient technique is proposed in this article to convert negative valued lumped elements in to practically realizable microstrip line structure.

This paper is organised into several sections. Section 2 discusses design, simulation and analysis of UWB LNA. The section 3 describes significant measurements and simulated results of the presented LNA. The section 4 emphasizes novelty and innovation of the article and section 5 comprises the conclusive remark of the designed work.

\section{UWB LNA DESIGN SIMULATION AND ANALYSIS}

This section explains the design of the UWB LNA design with the necessary steps involved in the design and simulation process. Moreover, it consists of following subsections as Complete Schematic, Layout, biasing topology, input and output negative value element matching circuit with realisation. Finally, it presents results which includes stability, noise figure, gain, input and output return.

Figure 1 shows the complete architecture of the design UWB LNA. The Avago technologies ATF36163 is a low-noise Psuedomorphic High Electron Mobility Transistor (PHEMT) has low noise-resistance, which reduces the sensitivity of noise performance. In Figure 1 netlist (ID=S1 and ID=S2) are s-parameters based model of ATF-36163 which is used for small signal analysis of the complete LNA design. Figure 1 includes many sub circuits namely "Input Dis Block", "Output dis network", "Drain Bias" and "Gate Bias" which will be discussed in further subsections with schematic and layout.

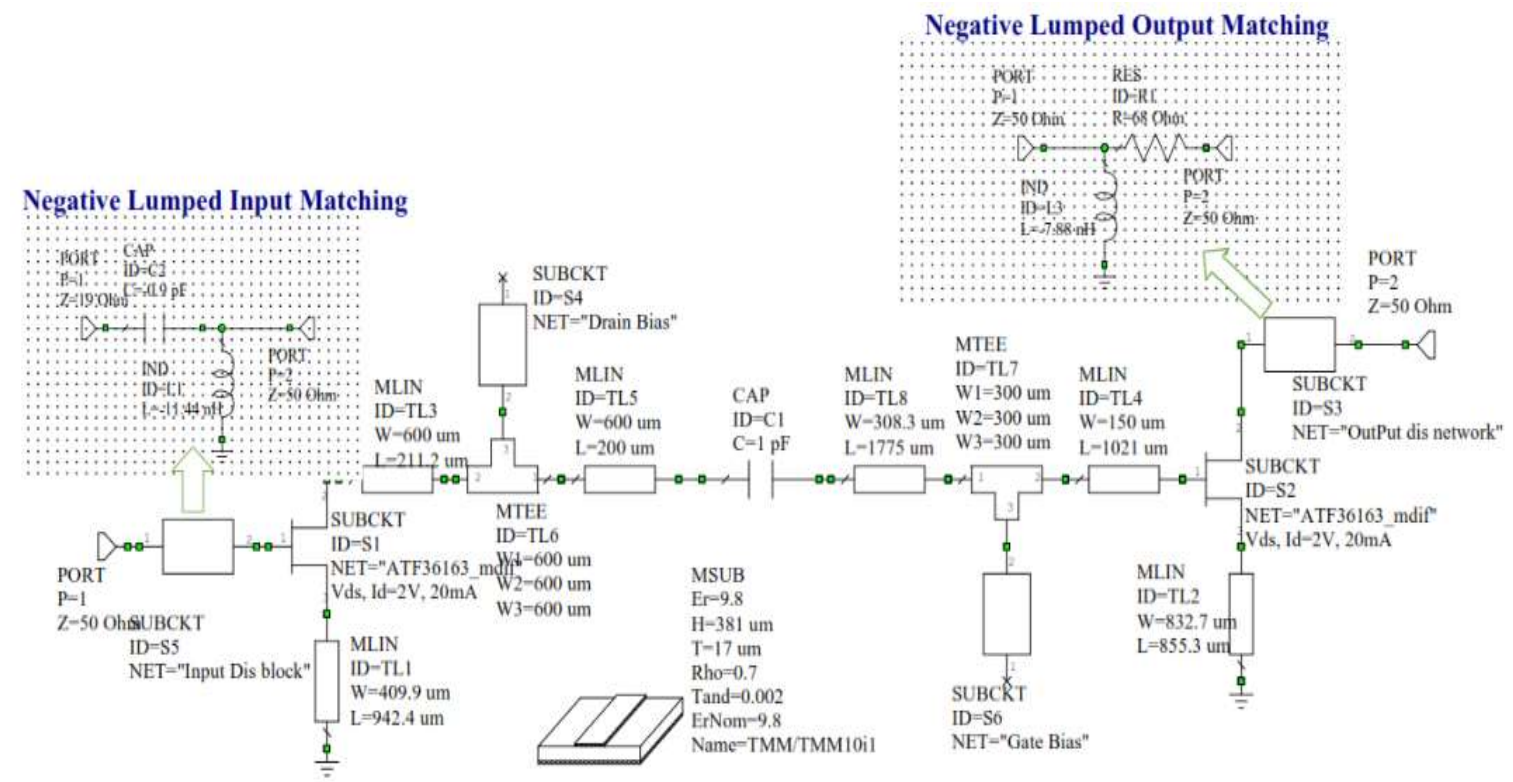

Figure 1. Circuit schematics of two stage UWB LNA

The sources are connected to ground through microstrip lines because the source degeneration is useful to insure stability and help the noise match [16]. The capacitor ( $\mathrm{ID}=\mathrm{C} 1$ ) is used for intermediate matching between two transistors and also to provide decoupling effects. The microstrip lines are used between every element throughout the design with dielectric constant $\mathrm{Er}=9.8$, height $\mathrm{H}=381 \mu \mathrm{m}$, conductor thickness $\mathrm{T}=17 \mu \mathrm{m}$, Metal bulk resistivity normalized to gold Rho=0.7 and Loss tangent of dielectric Tand $=0.002$. The initial design is carried out using negative lumped elements at both input and output matching of the design shows good LNA performance than the positive lumped elements in the circuit. So, the realization of the negative elements is discussed in the following sub sections. 


\subsection{Biasing network}

In this design, the drain and gate bias circuit are designed by using pair of inductor and bypass capacitors for pHEMT. The proper optimization of the lumped elements, microstrip lines with layout formation have ensured the LNA shows very low noise figure. This biasing network provides sufficient gain low noise figure and stable operation. Several experimentations are carried for the wideband LNA biasing circuits in the earlier published work [17-20].

\subsection{Realization of negative valued lumped elements}

This section explains the methodology to convert negative valued elements into practically realizable distributed elements. There are many methods to convert negative elements in to practically realizable circuit. Here, by using Richard's transform negative lumped elements are expressed in term of equivalent electrical length $(\beta 1)$. For the lossless transmission line telegrapher's equation, the propagation constant is purely imaginary $\gamma=j \beta$ is as follow:

$$
Z_{\text {in }}(\mathrm{l})=Z_{0} \frac{Z_{L}+\mathrm{j} Z_{0} \tan (\beta \mathrm{l})}{Z_{0}+\mathrm{j} Z_{L} \tan (\beta \mathrm{l})}
$$

where, wavenumber $\beta=\frac{2 \pi}{\lambda}$

While calculating $\beta$, the wavelength inside the transmission line with respect to microstrip line, dielectric material plays the vital role. In this realization method microstrip line geometry shown in Figure 2 is prepared by using series short stub (MLIN) and open stub (MLEF and MLSC). In case of shorted load $\left(Z_{L}=0\right)$, the input impedance will be purely imaginary and depends upon periodic variation of the wave number as shown in (2).

$$
Z_{\text {in }}(\mathrm{l})=\mathrm{j} Z_{0} \tan (\beta \mathrm{l})
$$

In case of open load $\left(Z_{L}=\infty\right)$, the input impedance will be again purely imaginary and depends upon periodic variation of the wave number as shown in (3).

$$
Z_{\text {in }}(\mathrm{l})=-\mathrm{j} Z_{0} \cot (\beta \mathrm{l})
$$

By the making use of above concepts, for the known value of the lumped elements $\mathrm{L}$ and $\mathrm{C}$, the electrical length can be calculated by the equations (4), (5) using Richard's transformation. From the basic equation, denoting the $\mathrm{L}$ and $\mathrm{C}$ as negative values, solving using by trigonometric techniques: By the making use of above concepts, For the known value of the lumped elements $\mathrm{L}$ and $\mathrm{C}$, the electrical length can be calculated by the equations (4), (5) using Richard's transformation. From the basic equation, denoting the L and $\mathrm{C}$ as negative values, solving using by trigonometric techniques:

$$
\begin{aligned}
& \frac{-j}{\omega(-C)}=-j Z_{0} \cot \beta l \\
& j \omega(-L)=j Z_{0} \tan \beta l
\end{aligned}
$$

Mathematical calculations are carried out for three different frequencies in order to find physical lengths of the distributed elements to the input matching network as shown in Figure 2. Figure 2 shows the schematic of the negative valued lumped elements for input matching of the LNA. The circuit is simulated initially by this network which ended with good performance of the LNA. To convert negative elements into distributed microstrip line calculation are made and depicted in Table 1.

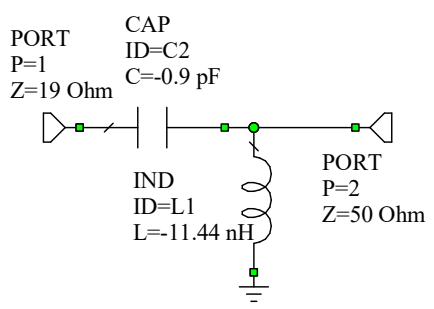

Figure 2. Negative valued lumped elements for input matching network 
Table 1 shows the calculated values of physical length and width of the microstrip lines whose $\mathcal{E}=9.8$, loss tangent Tand $=0.002$, conductivity $5.88 \times 10^{07} \mathrm{~s} / \mathrm{m}$, characteristics impedance $\mathrm{Z}_{0}=50 \Omega$, height $(H)=381 \mu \mathrm{m}$ and conductor thickness $(T)=17 \mu \mathrm{m}$. Since microstrip transmission line structure has metallization placed on top of a substrate and exposed to air, "effective" permittivity is a complex relationship between the substrate properties and air properties. To derive the frequency dependent (Keff) is done by using procedure suggested by Eikichi Yamashita, Mahmoud El Sabbagh and H.A. Atwater [17].

Table 1. Tabulated Frequency versus Physical Characteristics of Negative Valued Elements

\begin{tabular}{ccccc}
\hline & \multicolumn{3}{c}{ Negative valued lumped elements } & \multicolumn{2}{c}{$\mathrm{C}=-0.9 \mathrm{pF}$} \\
\hline Frequency in $\mathrm{GHz}$ & Physical Length $(\mu \mathrm{m})$ & Physical Width $(\mu \mathrm{m})$ & Physical Length $(\mu \mathrm{m})$ & Physical Width $(\mu \mathrm{m})$ \\
\hline 3 & 8425 & 354 & 4412 & 354 \\
6.5 & 4221 & 354 & 3092 & 354 \\
10 & 2796 & 355 & 2292 & 355 \\
$5.79 *$ & 4632 & 354 & 3150 & 354 \\
Optimized & 4736 & 300 & 3784 & 300 \\
\hline
\end{tabular}

*geometric mean, the numerical values which are presented here is based on theoretical concepts, which will have some degree deviate from the practical values with the various parasitic effects.

Microstrip line has excellent performance in the wideband characteristics and their size restricts for the utilization up to $10 \mathrm{GHz}$. The combination of the lumped and microstrip line will give optimistic performance of the LNA. Figure 3 shows the microstrip geometry with 'Short standard' (MLIN), 'open standard' (MLEF) and 'Shorted standard without end effect' (MLSC). The offset length of MLIN is arranged to build nearly for ideal reflection coefficients till $10 \mathrm{GHz}$ with polynomial coefficients of the negative capacitor. The length of the MLEF exhibits capacitances fringes and characterized by capacitance coefficients. The length of the MLSC exhibits the performance like negative inductor whose offset is analyzed without end effects.

Figure 3 shows the schematic of the distributed microstrip line prepared based on the values from the Table 1. Analysis is done with different frequencies for input matching circuit to calculate physical characteristics of the microstrip lines. The geometry structure is design to fulfill negative capacitor as linear series microstrip line and negative inductance as open microstrip line with end effect or shorted without end effects. All distributed elements like microstrip line exhibits frequency dependency and their characteristics introduce parasitic ripple effects in the wideband frequencies. It needs to construct geometric microstrip line structures in a such way that their frequency variation with S-parameters (especially S11( $\omega)$ ) conforms to a desirable function of $\omega$. The design of the microstrip line geometry to fulfill desired S-parameter performances is similar to filter realization. The determination of the filter pass band throughout the wideband characteristics is depend on the order of the filter. So, in this realization it is considered up to third order whose effective length is equal to $4736 \mu \mathrm{m}$ and average shunt open stubs length (with and without end effect) is $3784 \mu \mathrm{m}$.

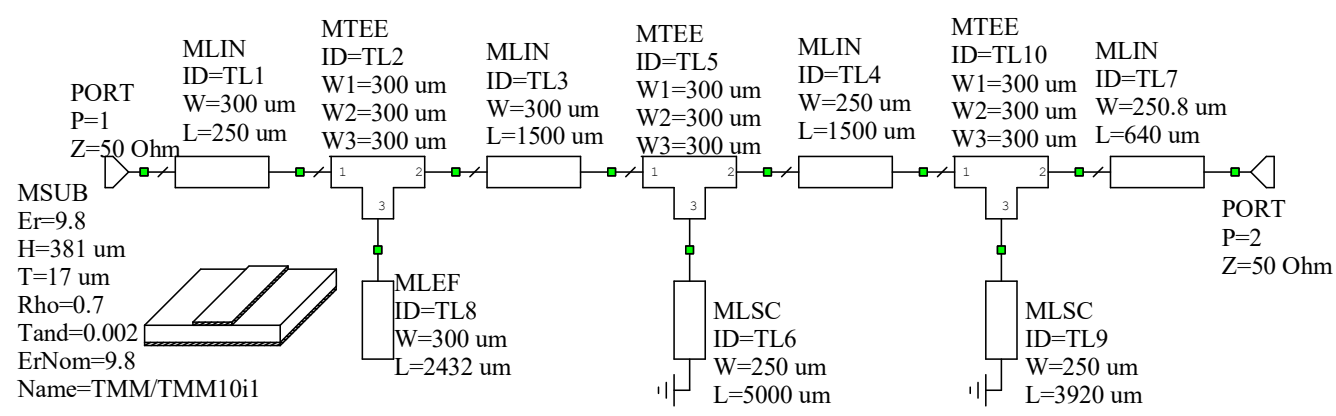

Figure 3. Schematic of the distributed elements of the input matching network

Figure 4 shows the 2D layout of the schematic shown in Figure 3. i.e. input matching network. For the consideration of the layout point of view all width of the microstrip line is kept constant of $300 \mu \mathrm{m}$ and corresponding lengths are optimized for required s-parameter characteristics. 


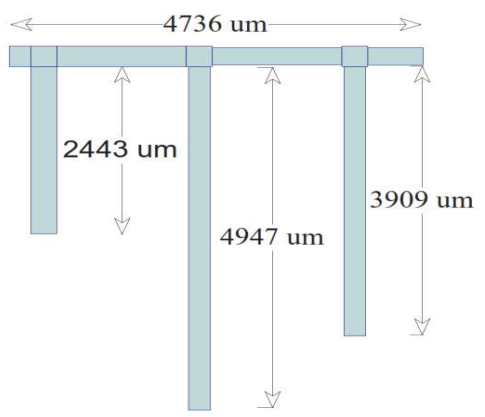

Figure 4. 2D layout of the distributed elements of the input matching network with to reference earlier figure

For ultra-wideband matching, a higher $\mathrm{Q}$ is needed or a higher order filter must be employed. Note that for higher $\mathrm{Q}$, the bandwidth gets narrower, requiring the filter to be tuned to compensate for mismatch and process variation. So, in this design experimentation is carried out up to $3^{\text {rd }}$ order Low pass filter microstrip line geometry for optimum bandwidth requirement. For the closed form expressions $Z_{0}$ and $\varepsilon_{\text {re }}$ when conductor thickness $\mathrm{t}=0$ is given by:

$$
Z_{0}=\frac{\eta}{2 \pi \sqrt{\varepsilon_{r e}}} \ln \left(\frac{8 h}{W}+0.25 \frac{W}{h}\right) \quad\left(\frac{W}{h}<1\right)
$$

where $\eta=120 \pi \Omega$ and $\varepsilon_{r e}=\frac{\varepsilon_{r}+1}{2}+\frac{\varepsilon_{r}-1}{2} F(W / h)$

$$
F\left(\frac{W}{h}\right)=(1+12 h / W)^{-\frac{1}{2}}+0.041(1-\mathrm{W} / \mathrm{h})^{2}
$$

The microstrip line negative capacitance (-C) and negative inductor (-L) is given per unit length with respect to $\mathrm{c}$ (speed of light).

$$
\begin{aligned}
& (-\mathrm{C})=\frac{\mathrm{Z}_{0} \sqrt{\varepsilon_{r e}}}{c} \\
& (-\mathrm{L})=\frac{\sqrt{\varepsilon_{r e}}}{\mathrm{Z}_{0} c}
\end{aligned}
$$

The bandwidth can be enhanced by using multiple sections of impedance transformed microstrip lines. It is important to consider the product of quality factor (Q) and Bandwidth (BW).

$$
\begin{aligned}
& Q * B W=\frac{2 \Gamma}{1-\Gamma^{2}} \quad \text { for single section } \\
& Q * B W=\frac{2 \sqrt{\Gamma}}{1-\Gamma} \quad \text { for double section } \\
& Q * B W=\frac{\pi}{\ln (1 / \Gamma)} \quad \text { for infinite section }
\end{aligned}
$$

For the ultrawide band matching, the reflection coefficient of the microstrip line geometry is given the series of sum of the partial reflection coefficient arising at the each discontinuous ' $L$ ' sections of the layout shown in the Figure 4. Where $\theta$ is electrical length separtion between multiple sections.

$$
\Gamma=\left|\Gamma_{1}\right|+\left|\Gamma_{2}\right| e^{-i 2 \theta}+\left|\Gamma_{3}\right| e^{-i 4 \theta}
$$

\subsection{S-parameter comparison of input matching}

Figure 5 shows the S11 characteristics of negative value lumped elements and microstrip line realization done using Richard's transform. The left axis represents the S11 characteristics of the negative lumped elements whose curve is linearly decreasing with respect to frequency. The right axis represents S11 characteristics microstrip line structure which is having minor variations with respect to frequency. 
Variations can be minimized by increasing the order of the microstrip line geometry. Similar technique is adopted to realize the microstrip line geometry from the negative valued elements of output matching network. Figure 6 shows the negative valued lumped elements used initially for output matching in the UWB LNA design.

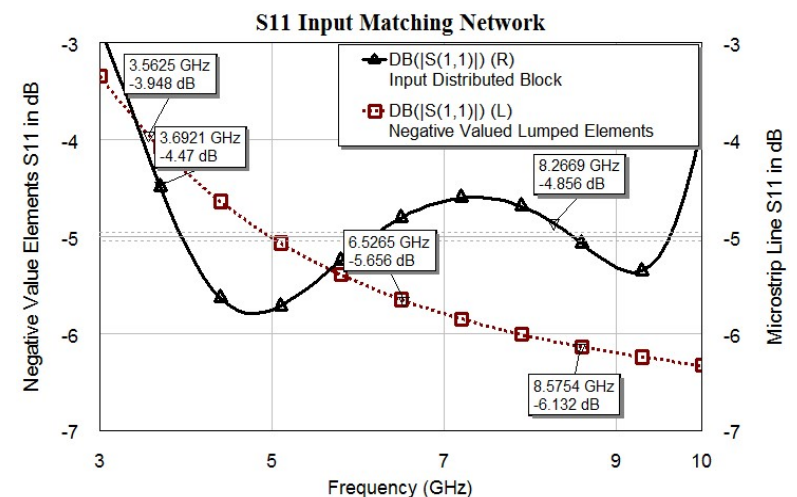

Figures 5. S11 parameter comparison between negative value elements and distributed elements structure of the input matching block

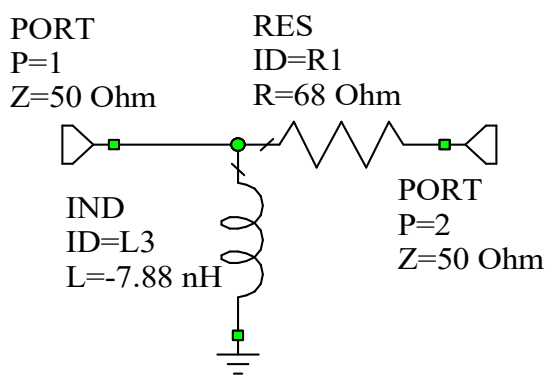

Figure 6. Negative valued lumped elements for output matching network

To compress the circuit and to avoid the complexity of the circuit, drain bias sub circuit is added inside the distributed element block of the output matching circuit which is clearly shown in the Figure 7. Same technique is applied here which were used in analysis of input matching block.

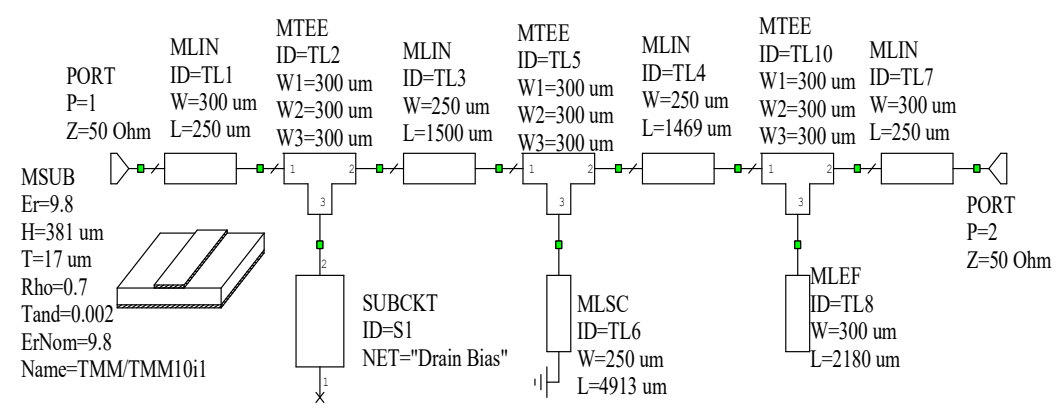

Figure 7. 2D layout of the distributed elements of the input matching network with reference earlier figure

\subsection{S-parameter comparison of output matching}

Figure 8 shows the S11 characteristics of negative value lumped elements and microstrip line realization done of the output network using Richard's transform. The left axis represents the S11 characteristics of the negative lumped elements whose curve is linearly decreasing with respect to frequency. The right axis represents S11 characteristics microstrip line structure which is having minor variations with respect to frequency. Variations can be minimized by increasing the order of the microstrip line geometry. 


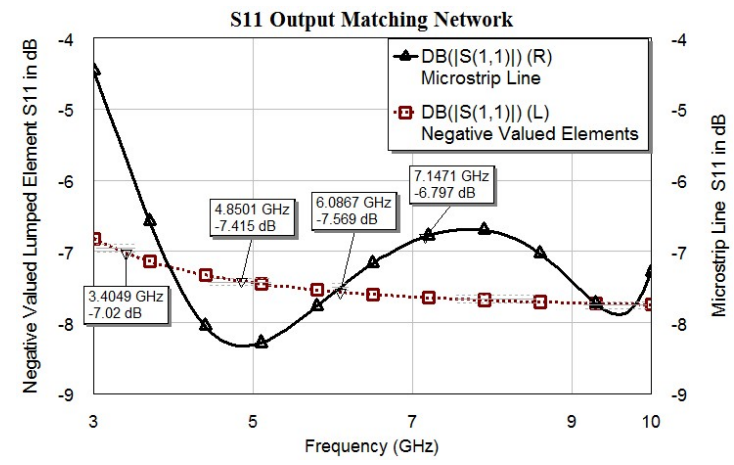

Figure 8. S11 parameter comparison between negative value elements and distributed elements structure of the input matching block

\section{RESULTS AND ANALYSIS}

The presented UWB LNA is designed by using ATF 36163 pHEMT transistors with negative image amplifier technique is measured with parameters like return loss, gain, noise figure and stability check. The following graphs with rectangular display type simulated results using AWR microwave tool are discussed in detail.

Figure 9 shows the simulated input return loss (S11) and output return loss (S22) of the complete UWB LNA by adopting the negative image amplifier technique. As experimented, the LNA is well-matched over the frequency range of interest. The circuit using negative lumped elements has achieved return losses less than $-10 \mathrm{~dB}$ without comprising other parameters of the LNA which was not achieved by using positive value lumped elements in the circuit. After the realization with microtrip line the input and output return loss values are less than $-8 \mathrm{~dB}$ throughout band. The return losses are deteriorating because of actual microstrip line technology cannot reach the same effects of negative valued lumped elements. To overcome this limitation, several experimentations are required to be carried out in conversion of negative elements into practically realizable circuits.

Figure 10 shows that the noise figure (NF) is below $2 \mathrm{~dB}$ from 3 to $10 \mathrm{GHz}$ and NFmin is less than $1.6 \mathrm{~dB}$ from 3 to $10 \mathrm{GHz}$. Another advantageous property of the negative image amplifier technique is that the gain, NF and return loss is obtained simultaneously. Indeed, the simulated NF almost equals the simulated NFmin of the complete LNA over a bandwidth from 4 to $10 \mathrm{GHz}$. The deviation between NF and NFmin is at lower frequencies occurred because of minor impedance mismatch at the input port. At low frequencies increase of NF is because flicker noise, whereas the reason for increase of NF at higher frequencies is drop in gain.

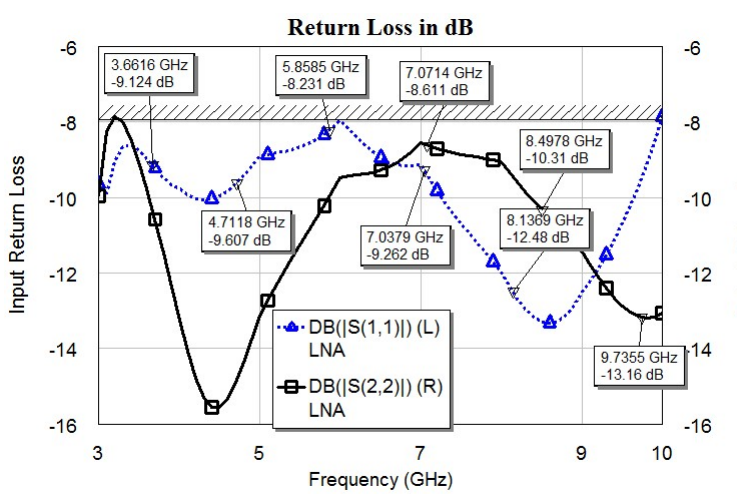

Figure 9. Return loss characteristics of the UWB LNA

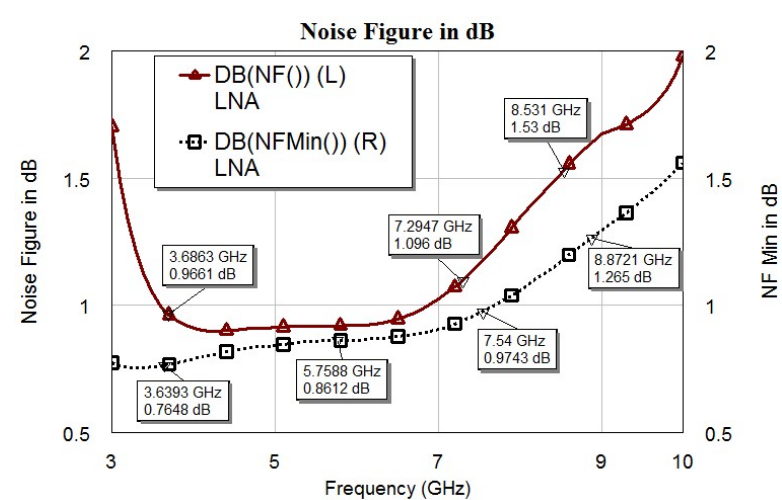

Figure 10. Noise figure and NFmin characteristics of the complete UWB LNA 
The noise sources and thermal noise voltages due to $R g$ and $R s$ respectively,

$$
F \min =1+\frac{2 \omega \operatorname{Cgs} \sqrt{R s+\frac{R g}{R i}}}{\mathrm{Gm}}
$$

Here, $\omega$ : angular frequency; Cgs: gate to source capacitance; Gm: transconductance; Rs: ohmic contact; Rg: metalization resistor and Ri: instrinsic resisance.

Figure 11 shows the gain characteristics of the designed UWB LNA. As for gain is concern, a sufficient high gain is required over complete UWB to withstand the effects noise figure of the upcoming stages in the receiver system. The transducer gain (S21) of this LNA is the resultant of two stage cascaded transistors is having variation in performance. If the gain flatness is required over UWB, an additional multiband gain peak in the following stages can be added to compensate gain drop in few sag. This case can be applied to this work 3-10 GHz UWB LNA. In order to make sure that designed UWB LNA is unconditionally stable, a rollet's factor $(\mathrm{K})$ test and the auxiliary factor $(\mathrm{B})$ are being plotted $[18,19]$. The $\mathrm{K}$-factor is depicted in left sided $\mathrm{Y}$-axis and secondary stability factor in right sided $\mathrm{Y}$ axis in the Figure 12, and the values of the $\mathrm{K}$-factor are greater than 1, B greater than zero over the entire $3-10 \mathrm{GHz}$ bandwidth all calculation are based on the s-parameters. In this consequence, the designed UWB LNA is made as unconditional stability with the help of microstrip line at the sources of the pHEMT.

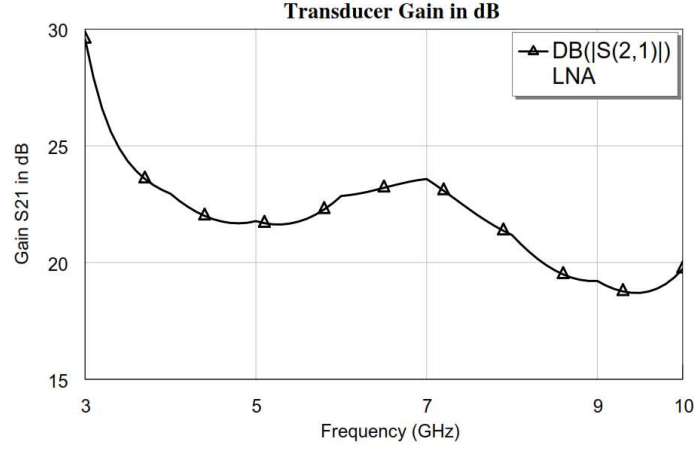

Figure 11. Gain performance of the UBW LNA

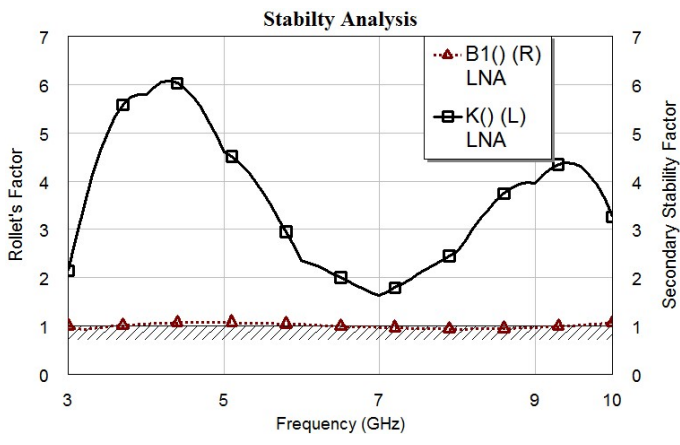

Figure 12. Stability test using K and B

Figure 13 displays the complete layout of UBW LNA on HMIC technology with all sub circuits i.e. Input and output matching blocks, drain and gate bias circuits, intermediate capacitors and transistors. Among the PCB layout, the red color rectangle component is the transistor, blue color lines all are microstrip lines and the rest drain and gate bias circuit compose of inductors and capacitors which shown soldier pads.

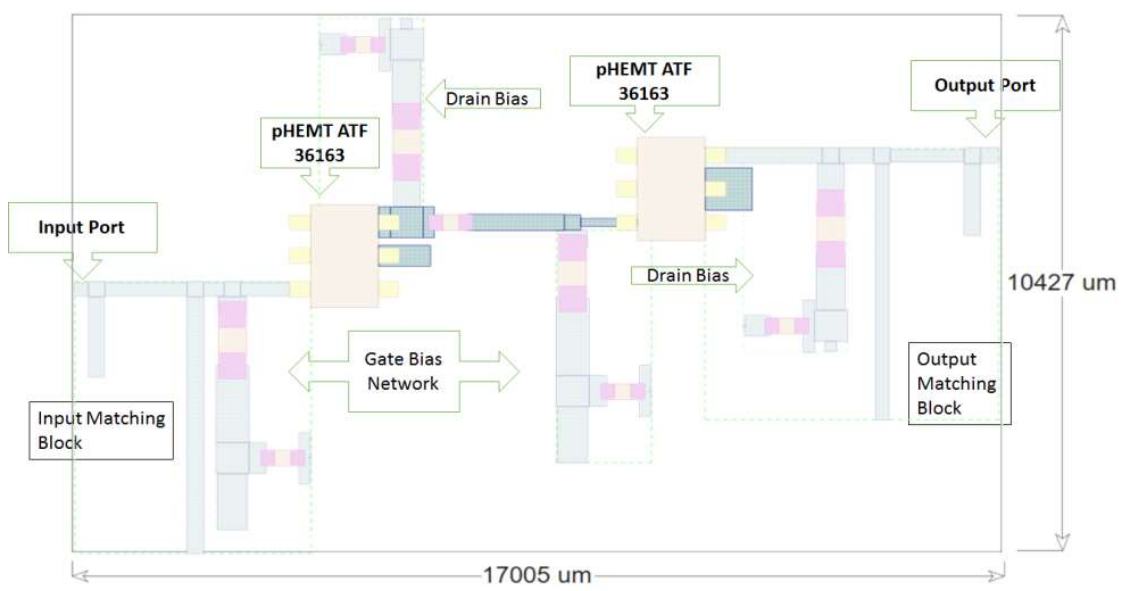

Figure 13. Two-dimensional layout of the complete UWB LNA which be fabricable 
The presented UBW LNA for 3-10GHz is summarized in the Table 2. The measured performances of the presented LNA in comparison with those of recently published LNAs. It is noted that the gain, bandwidth, noise figure and input return loss is compared clearly which shows the importance of this work.

Table 2. Performance Comparison with Recently Published Articles on LNAs

\begin{tabular}{ccccc}
\hline Reference & Frequency(GHz) & Gain in dB & Noise Figure in dB & S11 in dB \\
\hline [1] Zhengyu Sun & $0.7-5.2$ & $26-29$ & $2.6-4.4$ & $<-10$ \\
[2] Zulfa Hasan-Abrar & $1.5-6$ & $16-18$ & 1.5 & $<-5$ \\
[3] Khanhtran Phan & $1-12$ & $11-17$ & 2 & $<-10$ \\
[4] Chan-Sei Yoo & $2-11$ & 10 & 2 & $<-10$ \\
[5] sawarkar & $1.2-2.8$ & 25 & 0.5 & $<-8$ \\
This Work & $3-10$ & $>20$ & $<2$ & $<-8$ \\
This Work & $3-8.5$ & $22-23$ & $<1.5$ & $<-8$ \\
\hline
\end{tabular}

\section{INNOVATION IN THE PROPOSED WORK}

Though the negative image amplifier technique results in the performance improvement, yet it imposes the use of negative valued components which are not realizable. This technique presents a novel method of tackling the issue of the negative valued components. The conversion of negative reactance into realizable component is done using microstrip line structure. The complete strategy leads to realizable design of LNA circuitry. This approach provides following remarkable achievements of negative image amplifier technique over conventional matching circuit:

a. Compressed and squeezed down to a smaller size, total board outline is $17 \mathrm{~mm} \times 10.5 \mathrm{~mm}$

b. Sufficient gain is achieved with unconditional stability.

c. Good noise figure less than $2 \mathrm{~dB}$ over complete $3-10 \mathrm{GHz}$ band

d. Good bandwidth 3-10 GHz, which is ultra-wideband with return loss of $-8 \mathrm{~dB}$.

\section{CONCLUSION}

This paper proposes novel procedure of acknowledgment of the negative valued lumped elements by utilization of the microstrip line parasitic effects. Whose effects is clearly explained with supporting mathematical analysis and simulation results. Bandwidth characterizing for the LNA is very complex, this work has met with several conflicting measurements like return loss, NF, Gain and stability based on the S-parameter analysis. This work presented the two-stage cascaded LNA which exhibits average gain of $23 \mathrm{~dB}$ gain and low noise figure of less than $2 \mathrm{~dB}$ with return loss less $-8 \mathrm{~dB}$ for UWB 3-10GHz. The designed LNA furnished least NF alongside with good gain when contrasted with recent works. Consequently, the coveted focus of the design was achieved with a specific end goal to be incorporated as a first stage of the receiver's circuitry.

\section{ACKNOWLEDGEMENTS}

This work is upheld and maintained by MCT's Rajiv Gandhi Institute of Technology, Mumbai. Moreover, authors might need to express appreciation towards Dr. Udhav Bhosle, Principal of RGIT for basic support and comfort for this investigation work. The authors may need to one of a kind an obligation of appreciation is all together for Mr. Manjunatha Reddy H. V General Manager, RF and $\mu \mathrm{W}$ division, Icon Design Automation Pvt. Ltd.

\section{REFERENCES}

[1] Z. Sun, H. Yang, L. Zhang and Y. Yan, "Low Noise Fully Single-Ended Broadband Receiver Front-End with InGaAs pHEMT Technology," 2013 IEEE International Wireless Symposium (IWS), Beijing, 2013, pp. 1-4.

[2] Z. Hasan-Abrar, Y. H. Chow and Y. W. Eng, "A Low-voltage, Fully-integrated (1.5-6) GHz Low-Noise Amplifier in E-mode pHEMT Technology for Multiband, Multimode Applications," 2008 European Microwave Integrated Circuit Conference, Amsterdam, 2008, pp. 306-309.

[3] K. Phan, J. Kessler, H. Morkner, M. Vice, L. Nguyen and J. Roland, "A Miniaturized Wafer-Scale Package Demonstrated with Three Enhancement Mode Amplifiers," 2008 European Microwave Integrated Circuit Conference, Amsterdam, 2008, pp. 48-51.

[4] C. S. Yoo et al., "A Ultrawideband Low Noise Amplifier Using Metamorphic HEMT on Organic Substrate," 2008 Asia-Pacific Microwave Conference, Macau, 2008, pp. 1-4. 
[5] K. G. Sawarkar, R. V. Navagare and K. Tuckley, "Bandwidth Enhancement of Low Noise Amplifier Using Inductive Source and Distributed Components," 2016 IEEE International Conference on Recent Trends in Electronics, Information \& Communication Technology (RTEICT), Bangalore, 2016, pp. 1461-1465.

[6] A. K. Ray and R. C. Shit, "Design of Ultra-Low Noise, Wideband Low-Noise Amplifier for Highly Survival Radar Receiver," in IET Circuits, Devices \& Systems, vol. 10, pp. 473-480, 2016.

[7] P. Choi et al., "A 5.9-GHz Fully Integrated GaN Frontend Design With Physics-Based RF Compact Model," in IEEE Transactions on Microwave Theory and Techniques, vol. 63, pp. 1163-1173, April 2015.

[8] G. A. Ellis et al., "Wideband AlGaN/GaN HEMT MMIC Low Noise Amplifier," 2004 IEEE MTT-S International Microwave Symposium Digest (IEEE Cat. No.04CH37535), vol.1, pp. 153-156, 2004.

[9] G. Nikandish, A. Yousefi and M. Kalantari, "A Broadband Multistage LNA With Bandwidth and Linearity Enhancement," in IEEE Microwave and Wireless Components Letters, vol. 26, pp. 834-836, Oct. 2016.

[10] A. R. A. Kumar, A. Dutta and S. G. Singh, "Transformer Coupled Novel Noise Cancellation Technique for Subthreshold UWB LNA," 2012 5th International Conference on Computers and Devices for Communication (CODEC), Kolkata, 2012, pp. 1-4.

[11] H. Cruz, S. Y. Lee and C. H. Luo, "A 3-to-7GHz wideband LNA with IIP3 of $-2 \mathrm{dBm}$ and $0.5 \mathrm{~dB}$ in-band gain ripple," 2015 IEEE International Wireless Symposium (IWS 2015), Shenzhen, 2015, pp. 1-4.

[12] C. T. Fu, C. N. Kuo and S. S. Taylor, "Low-Noise Amplifier Design with Dual Reactive Feedback for Broadband Simultaneous Noise and Impedance Matching," in IEEE Transactions on Microwave Theory and Techniques, vol. 58, pp. 795-806, April 2010.

[13] Pramod K B Rangaiah, Kumaraswamy H V; A 1-5Ghz, Hybrid Mic Wideband LNA utilizing Microstrip Geometric Structure Variety for Performance Improvement, Transactions on Networks and Communications, vol 5, pp: 15-31, April 2017.

[14] J. Y. Lee, H. K. Park, H. J. Chang and T. Y. Yun, "Low-power UWB LNA with Common-gate and Current-reuse Techniques," in IET Microwaves, Antennas \& Propagation, vol. 6, pp. 793-799, May 2012.

[15] H. Ham, J. y. Lee and T. y. Yun, "21-dB Gain Ultra-wideband Complementary Metal-oxide Semiconductor Lownoise Amplifier with Current-reuse Technique," in IET Microwaves, Antennas \& Propagation, vol. 5, no. 12, pp. 1495-1501, September 162011.

[16] L. Pantoli, A. Barigelli, G. Leuzzi and F. Vitulli, "Analysis and Design of a Q/V-band Low-noise Amplifier in GaAs-based $0.1 \mu \mathrm{m}$ pHEMT Technology," in IET Microwaves, Antennas \& Propagation, vol. 10, no. 14, pp. 1500-1506, 11192016.

[17] H. A. Atwater, "Tests of Microstrip Dispersion Formulas," in IEEE Transactions on Microwave Theory and Techniques, vol. 36, no. 3, pp. 619-621, Mar 1988.

[18] Pozar, D.M, Microwave engineering, Wiley, New York, 1998.

[19] Edwards, M.-L., Sinsky, J.-H., "A New Criteria for Linear 2-port Stability Using a Single-geometrically Derived Parameter," IEEE Trans. Microw. Theory Tech., vol. 40, pp. 2083-281, 1992.

[20] Sawarkar, Kishor G., Kushal Tuckley, K. B. Pramod, and H. V. Kumaraswamy. "The Approach on Influence of Biasing Circuit in Wideband Low Noise Amplifier to Evaluate Robustness Performance." Empirical Research Press Ltd. (2017): 60

\section{BIOGRAPHIES OF AUTHORS}
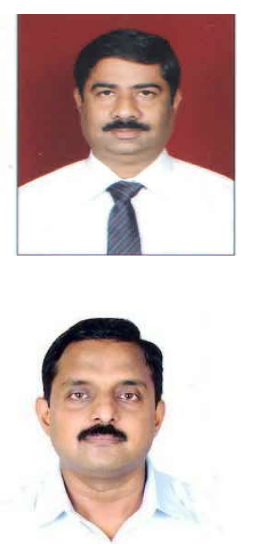

K. G. Sawarkar is working towards Ph.D. as Research Scholar at MCT's Rajiv Gandhi Institute of Technology, Mumbai University. Currently working as teaching academician in the department of Electronics \& Telecommunication Engineering. His Area of Interest is Design of analog circuits, RF circuits and systems He has done his BE in Electronics under Amravati University and M.E in Electronics from Mumbai University.

E-mail :kishor.sawarkar@mctrgit.ac.in, sawarkarkishor@gmail.com

Dr. Kushal R. Tuckley is currently working as Chairman. Head, R\&D, AGV systems pvt Ltd. Mumbai. He is an expert in the field of DSP, Radars and RF/Microwave systems. He has B. Tech, M.Tech and PhD from IIT Bombay. He had a 25 years long career as a scientist in Society for Applied Microwave Electronics Engineering and Research (SAMEER), a premium research institute in microwave systems engineering. He has significant contribution in many of the prestigious projects commissioned by the Govt. of India. He had a stint at Astra Microwave Products Ltd.

E-mail: kushaltuckley@agv-systems.in,kushal@ee.iitb.ac.in 